\title{
Accelerated Snowmelt Protocol to Simulate Climate Change Induced Impacts on Snowpack Dependent Ecosystems
}

\author{
Laura T. Leonard ${ }^{1}$, Chelsea Wilmer ${ }^{2}$, Heidi Steltzer ${ }^{3}$,
} Kenneth $\mathrm{H}$. Williams ${ }^{2,4}$ and Jonathan O. Sharp ${ }^{1,5, *}$

\begin{abstract}
${ }^{1}$ Department of Civil and Environmental Engineering, Colorado School of Mines, Golden, CO, United States; ${ }^{2}$ Rocky Mountain Biological Laboratory, Gothic, CO, United States; ${ }^{3}$ Department of Environmental Biology, Fort Lewis College, Durango, CO, United States; ${ }^{4}$ Lawrence Berkeley National Laboratory, Berkeley, CA, United States; ${ }^{5}$ Hydrologic Science and Engineering Program, Colorado School of Mines, Golden, CO, United States

*For correspondence: jsharp@mines.edu
\end{abstract}

\begin{abstract}
[Abstract] Field studies that simulate the effects of climate change are important for a predictive understanding of ecosystem responses to a changing environment. Among many concerns, regional warming can result in advanced timing of spring snowmelt in snowpack dependent ecosystems, which could lead to longer snow-free periods and drier summer soils. Past studies investigating these impacts of climate change have manipulated snowmelt with a variety of techniques that include manual snowpack alteration with a shovel, infrared radiation, black sand and fabric covers. Within these studies however, sufficient documentation of methods is limited, which can make experimental reproduction difficult. Here, we outline a detailed plot-scale protocol that utilizes a permeable black geotextile fabric deployed on top of an isothermal spring snowpack to induce advanced snowmelt. The method offers a reliable and cost-effective approach to induce snowmelt by passively increasing solar radiation absorption at the snow surface. In addition, control configurations with no snowpack manipulation are paired adjacent to the induced snowmelt plot for experimental comparison. Past and ongoing deployments in Colorado subalpine ecosystems indicate that this approach can accelerate snowmelt by 14-23 days, effectively mimicking snowmelt timing at lower elevations. This protocol can be applied to a variety of studies to understand the hydrological, ecological, and geochemical impacts of regional warming in snowpack dependent ecosystems.
\end{abstract}

Keywords: Biogeochemistry, Paired field studies, Climate warming, Induced early snowmelt, Ecosystem, Plant phenology

[Background] The intergovernmental panel on climate change reported in 2014 that over a period of 1976-2012 snow cover in the Northern Hemisphere has decreased by $11.7 \%$ per decade in the month of June (IPCC, 2014). Snowpack storage is crucial for headwater regions, and early water release could lead to water resource management and ecosystem function issues (Elias et al., 2015; Demaria et al., 2016). It has been predicted that snowmelt timing in the western U.S. could be enhanced by as much as two months by the end of this century (Rauscher et al., 2008), while a more recent study (Clow et al., 2016) reported advanced snowmelt offset by $1-2$ weeks over the past two decades in Colorado, which 
Please cite this article as: Leonard et. al., (2020). Accelerated Snowmelt Protocol to Simulate Climate Change Induced Impacts on Snowpack Dependent Ecosystems,Bio-protocol 10 (6): e3557. DOI: 10.21769/BioProtoc.3557.

is an essential headwater state. Shorter periods of snow cover and earlier water release have the potential to affect several aspects of ecosystem health in mountainous environments such as water availability and storage (Barnett et al., 2005), plant phenology and succession (Livensperger et al., 2016), and the soil microbial community structure (de Vries and Griffiths, 2018). With several aspects of the environment impacted by shifts in snowmelt timing, it is helpful to have a reliable and reproducible method that enables paired comparisons of enhanced and natural snowmelt across researchers and geographic regions.

A variety of methods have been used to manipulate snowmelt rates. Some have used a shovel to remove snow depth (Wipf et al., 2006), while others implemented more involved methods such as infrared heating sources (Harte et al., 1995; Bokhorst et al., 2008), or distributing black sand across the snow surface area (Steltzer et al., 2009; Blankinship et al., 2018). In contrast to these methods, the protocol described here is a noninvasive, low cost, and easily reproducible method that increases the absorption of solar radiation with a permeable black fabric deployed directly on the snow surface (Walsh et al., 2003; Steltzer et al., 2009). In addition, the method enables paired contrasts between accelerated and natural snowmelt processes in adjacent plots with conserved water equivalents. In contrast to black sand applications, the fabric is removed once the snow has fully melted and does not leave residual non-native material in associated soils. This refined protocol builds upon insights from past studies using permeable fabric covers (Steltzer et al., 2009) with a goal of increasing method utility, adoption and reproducibility. More recent efforts from 2017-2019 in Crested Butte, Colorado have documented snowmelt advancement of 14-23 days when contrasting manipulated and control plots at an elevation of 3,170 meters (Figure 1). The protocol and equipment explained below allows others to establish, deploy and monitor several aspects of the snowmelt process and can be adapted for a variety of environmental studies including but not limited to plant litter decay processes, geochemical shifts in the hydrosphere, and local plant phenology and growth.

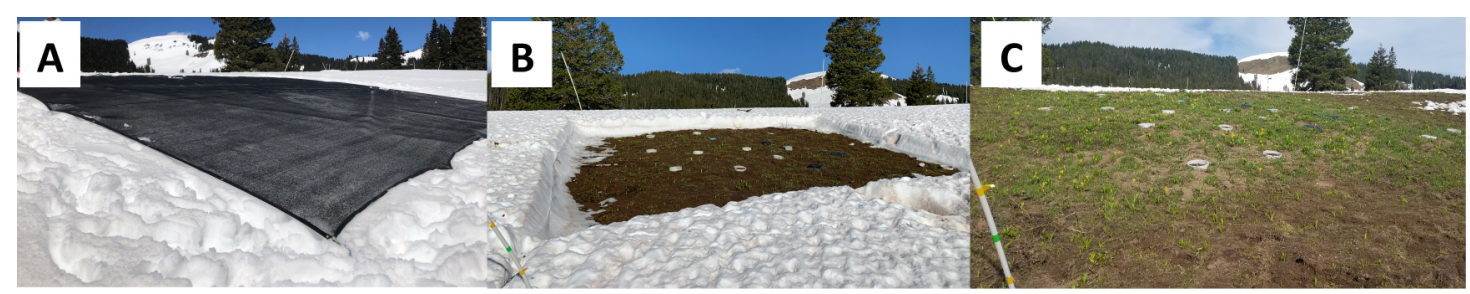

Figure 1. Visual representation of tarp deployment and resulting accelerated snowmelt during spring 2019 manipulation. A. The geotextile fabric was deployed after an isothermal snowpack was established. B. Removal of the tarp reveals the clear influence of this protocol on enhanced melting in the target plot. C. Contrast of the accelerated snowmelt plot with the control snowmelt reveals localized, accelerated onset of plant growth in the manipulated plot. Note, white rings visible within the area of manipulation were used for a separate experiment and are not discussed in this protocol $(B, C)$. 


\section{Materials and Reagents}

A. Snow cover and anchors

1. Permeable black UV PE Knitted Shade Cloth $50 \%: 9.8$ × $9.8 \mathrm{~m}$ with hemmed grommets spaced every $1.2 \mathrm{~m}$ (Agriculture Solutions, catalog number: KS50)

2. Type III 550 paracord $60 \mathrm{~m}$ spool (Paracord Planet)

3. 12 Pack aluminum alloy locking Carabiners (Bondream)

4. Four $1.1 \mathrm{~cm} \times 15.2 \mathrm{~cm}$ wooden dowels (Hardware Store)

5. Four $5 \mathrm{~cm} \times 10 \mathrm{~cm} \times 12 \mathrm{~cm}$ wood blocks (Hardware Store)

6. Drill with a $6.35 \mathrm{~mm}$ bit (Hardware Store)

B. Snow stakes and plot markers

1. Four PVC pipes $2.5 \mathrm{~cm} \times 3 \mathrm{~m}$; Plain end PVC Schedule 40 Pressure Pipe (Hardware Store)

2. Multi-Colored Duct Tape (Hardware Store)

3. Four $1.3 \mathrm{~cm} \times 1.2 \mathrm{~m} \# 4$ Rebar (Hardware Store)

4. Small sledge hammer (Hardware Store)

\section{Equipment}

A. Deployment

1. Post hole digger or metal shovel (Hardware Store)

2. $35.5 \mathrm{~cm}$ Big Grip Garden Knife (Hardware Store)

B. Data analysis

1. Two Hobo H21 Data Loggers (Onset HOBO data logger, catalog number: $\mathrm{H} 21-002$ )

2. Four Hobo moisture probes (Onset $\mathrm{HOBO}$ data logger, catalog number: S-SMD-M005)

3. Four Hobo temperature probes (Onset HOBO data logger, catalog number: S-TMB-M006)

4. Two Chemical resistant washdown enclosures $22.9 \mathrm{~cm} \times 22.9 \mathrm{~cm} \times 11.4 \mathrm{~cm}$ (McMaster-Carr, catalog number: 8261k27)

5. Ultra-abrasion resistant expandable sleeving stainless steel $1.3 \mathrm{~cm} \mathrm{ID,} 3 \mathrm{~m}$ length (McMasterCarr, catalog number: 1478T3)

6. $250 \mathrm{~g}$ Plumber's putty (Hardware Store)

7. TimelapseCam Pro camera (Wingscapes, catalog number: WCB-00121)

8. At least 6 Lysimeters ( $15.2 \mathrm{~cm}$ Soil Moisture lysimeters, catalog number: 1905L06) 


\section{Procedure}

A. Plot Selection: During fall snow-free season

1. Find a location for experimentation with annual snowfall that allows for snowpack manipulation: Ensure the location is not heavily forested or shaded and has a slope aspect that receives direct sunlight radiation throughout the day. In addition, seasonal human and animal traffic should be considered. It is possible that snowmobilers and skiers may frequent the area and precautions may be necessary to prevent damage to the plot. In our case, the PVC snow stakes were successful in designating the area and prevented most human interference.

2. The control and manipulated plots should be positioned horizontally adjacent at the same elevation and slope aspect to allow for comparison while also preventing interference between the two plots.

B. Snow fabric preparation: During fall snow-free season

1. Choose a geotextile fabric surface area larger than the predetermined plot perimeter under investigation to allow for error in deployment placement as well as to minimize boundary effects. Here, we utilized a $9.8 \times 9.8 \mathrm{~m}$ geotextile that was deployed over a $7.6 \times 7.6 \mathrm{~m}$ experimental grid (Figure 2).

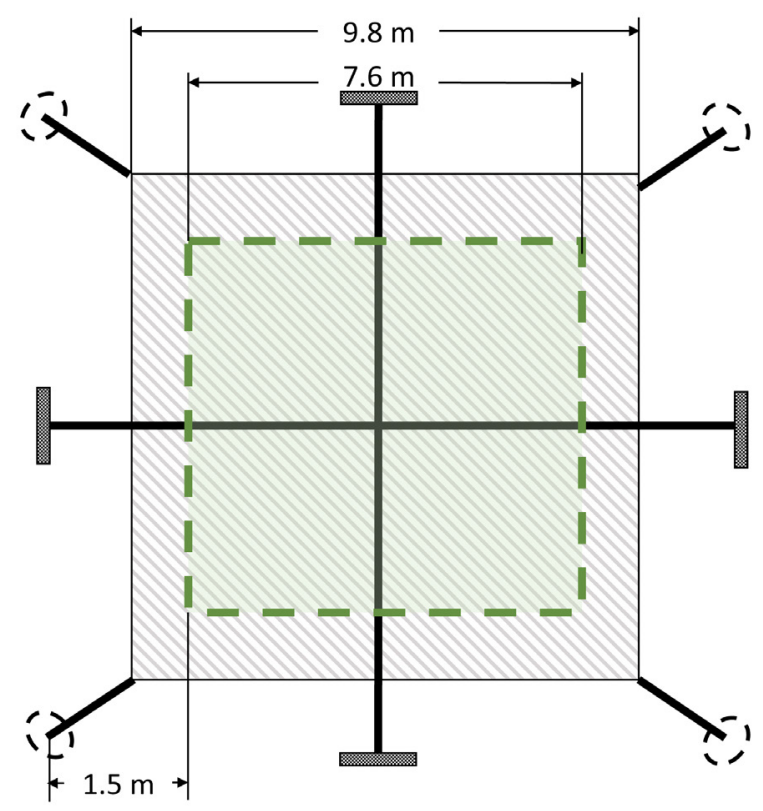

Figure 2. Geotextile fabric schematic according to our plot dimensions. Perimeter sizing is easily customizable for different plot sizes. Here our plot dimensions are shown with a square $7.6 \times 7.6 \mathrm{~m}$ plot (green dashed square), and a $9.8 \times 9.8 \mathrm{~m}$ geotextile fabric (larger shaded square). The rope lengths should be long enough (at least $1.5 \mathrm{~m}$ from the plot) to maintain sufficient tension over the desired plot while allowing the fabric to stay flush against the snow surface area. Carabiners are shown at the fabric corners (dashed ovals) and dowels are shown 
tied to the ropes threaded across the middle length and width of the fabric (rectangles). Note dowels, rope and carabiner objects are enlarged for visual purposes and are not to scale.

2. Cut four $0.6 \mathrm{~m}$ lengths of paracord and thread each through the grommets of the four corners of the fabric and tie the ends to a carabiner. These ends will serve as attachments to the corner snow anchors described in Procedure $\mathrm{C}$ and as shown in Figure 2.

3. Cut two lengths of paracord that will thread across the full length and width of the geotextile fabric as shown in Figure 2. For our experimental area, two $13 \mathrm{~m}$ lengths of rope were cut. When choosing a length, ensure enough extra paracord ( 1-1.5 $\mathrm{m})$ is allowed on each end of the tarp to anchor the fabric and allow tension during snowmelt for the fabric to fall flush with the melted snow surface.

4. Drill holes in the middle of the wooden dowels with a $6.35 \mathrm{~mm}$ or larger drill bit to ensure the paracord will thread through the hole.

5. Thread one stretch of the pre-cut paracord along the length and the other along the width of the fabric through the middle edge grommets and tie the ends of the paracord through the drilled holes of the wooden dowels (Figure 2).

6. Determine the logistics of deployment and if it is feasible to leave the geotextile fabric folded nearby the plot under study to be recovered during deployment. If not, plan to travel with the tarp during deployment. In our case, we traveled with the snow fabric tied to a travel pack.

C. Snow stake deployment: During fall snow-free season

1. Prepare the snow stakes. In our case we used a $3 \mathrm{~m}$ PVC pipe length; however, if a higher snowpack is expected the PVC pipe may need to be longer than documented here. Mark each sequential height of one foot with multicolored duct tape on each pipe to allow for approximate snow depth measurements. Multicolored tape is suggested to ensure ease of measuring sequential heights from the time-lapse photos.

2. Prepare snow anchors: drill holes in the middle of the wooden blocks with a $6.35 \mathrm{~mm}$ or larger drill bit to ensure the paracord will thread through.

3. Drill a hole at the bottom and top of the PVC pipes on the same side approximately $15 \mathrm{~cm}$ from the ends using a $6.35 \mathrm{~mm}$ or larger drill bit.

4. Thread the paracord through the drilled hole at the top of the PVC pipe, tying it securely at the top of the pipe. Thread the tied portion of paracord along the outside of the pipe and into the bottom drilled hole and out through the bottom of the pipe leaving $0.3-0.6 \mathrm{~m}$ of free paracord.

5. Tie the free paracord end to the wood block through the drilled hole and do this for all four PVC pipes (Figure 3). 


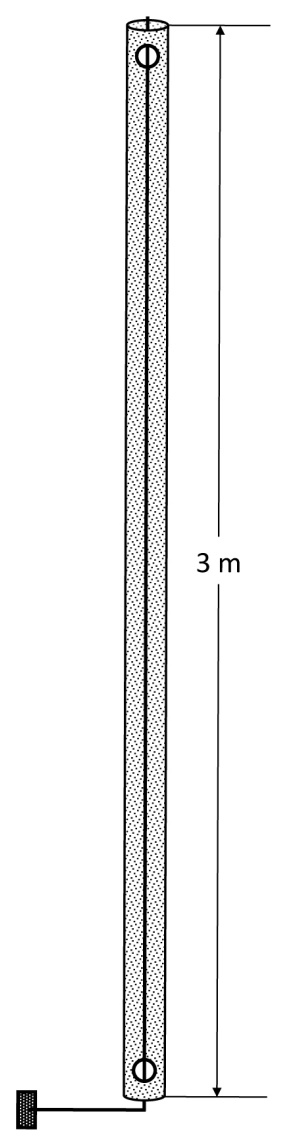

Figure 3. Snow stake schematic. The anchoring design is shown with holes (circles) drilled in the PVC pipe with paracord (black line) threaded along the height of the pipe to the bottom. The drilled hole at the top of the pole enables quick access to the paracord when the snow has fallen, and the bottom hole is an attachment for the wood block to serve as a snow anchor (bottom rectangle).

6. Determine the desired snowmelt area by providing at least $1.5 \mathrm{~m}$ extra distance from the perimeter of the plot under investigation for anchors to be placed to secure the fabric (Figure 2). Hammer the rebar into the four corners of the determined anchor perimeter until half of the rebar is above the soil surface.

7. Place the PVC pipes over each rebar with the bottom of the pipe flush against the soil surface to stand in an upright position during the snow season. Ensure the wood block is located at the bottom of the PVC pipe.

8. Extend the wooden block at the bottom of the PVC pipe outward from the plot to serve as a snow anchor during snowfall.

D. Data logger assembly: During fall snow-free season

1. Prepare the soil moisture and temperature probes for the induced snowmelt and control plots following manufacturer instructions. You should have at least two of each probe placed in the center of both plots to ensure redundancy. 
2. Avoid leaving loose wires open to the environment by threading the probe wires through the abrasion resistant steel covers for resistance against critters.

3. Use the garden knife to make a cut into the soil for the temperature $(6 \mathrm{~cm}$ depth) and moisture probes (6-12 cm depth) and insert the sensors into each cut.

4. Place the data logger in the respective waterproof enclosure for each plot to avoid weather damage. You will need to drill a hole in one side of each box to allow the sensor wires to thread through into the box. Once the sensor wires are threaded through, use plumber's putty to seal the drilled hole.

5. Connect the sensors to the data logger station using manufacturer instructions and ensure your computer is connected correctly to the logger using the Hoboware software provided. Set the logger to begin logging hourly data acquisition.

6. Find a landmark such as a tree nearby to secure the time-lapse camera, or use a tripod to capture snowmelt progression directly above the plots. Ensure the camera is higher than the expected snowpack height to ensure photos are collected daily.

7. Set the camera to activate daily time series using manufacturer instructions. In our case, the time series was set for collection every $24 \mathrm{~h}$ in the afternoon.

E. Snow fabric deployment: During spring peak snowpack

1. Determine the best time frame for deployment: Ideally the snowpack will be isothermal and entering a net seasonal melting cycle. Our deployment was conducted between April 10-25 in 2017-2019 in Colorado at 3,170 m.

2. It is best to determine a time when peak snowpack is reached, and no further large snow storms are forecasted. Over the years 2017-2019 snowpack during deployment ranged 1.2-1.5 m.

3. Access to the site can vary depending upon conditions and distance. Snowshoes or backcountry skis work well with minimal damage to the site; however, snowmobile access is fastest for longer deployment distances.

4. Once at the site with the pre-deployed snow stakes identified, lay down the prepared fabric with the threaded paracord facing upward and accessible.

5. Align the corners of the fabric with the snow stake anchor points. Untie the pre-deployed paracord from the top of the snow stakes and tie each tight to the carabiners that are attached to the corner grommets of the fabric (Figure 4A). Note that the end of the untied paracord will be buried in the snow at the bottom of the snow stake.

6. Dig a trench of increasing depth in the snow from the corners of the geotextile along the length of the paracord to achieve roughly a 45-degree angle to ensure tension on the corners as the snow melts.

7. Extend the length of the paracord attached to the grommets at the middle length and width of the fabric to secure the four middle edges with the wooden dowels (Figure 4B).

8. Dig a tapered trench of increasing depth in the snow under the paracord from the edge of the fabric to the end where the wooden dowels will be buried to maintain a 45-degree angle. Use 
the garden knife to lead the tapered trench and use the post hole digger to dig a hole at the deepest depth of the snow. For our deployments, a tapered trench that reached a $1.2 \mathrm{~m}$ deep hole 1-1.5 $\mathrm{m}$ from the fabric edge was sufficient.

9. Bury the dowels by placing the dowel at the bottom of each hole and fill the holes with snow.

10. Ensure the fabric is flush to the snow surface and secure at all four corners and middle lengths of the perimeter (Figure 4C).

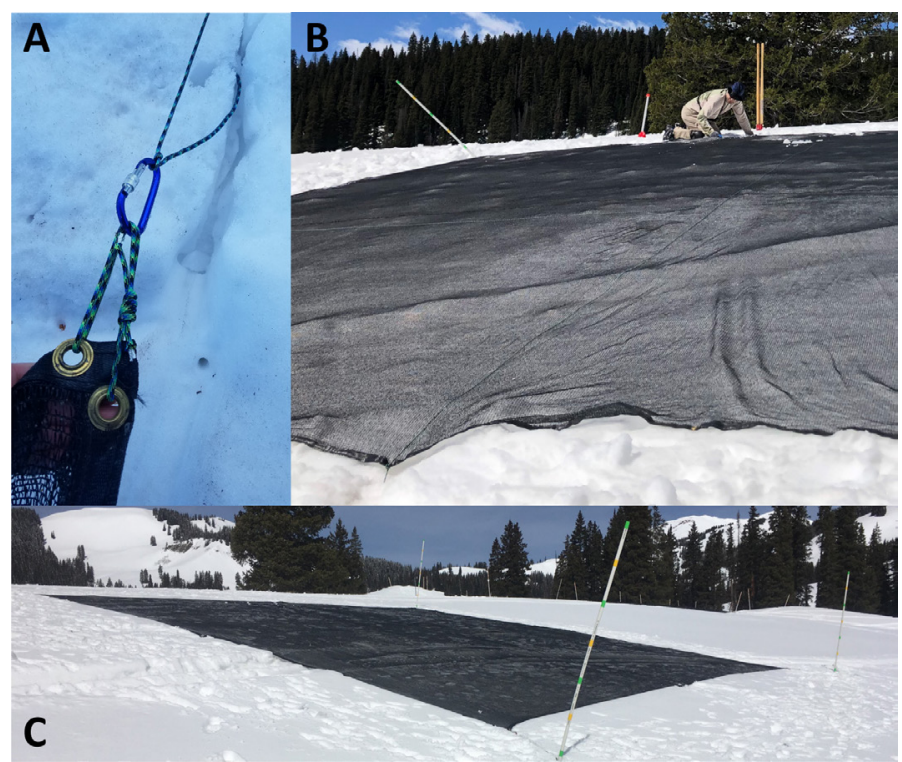

Figure 4. Geotextile fabric after a successful deployment. A. The corners of the geotextile fabric are tied securely to a carabiner and the paracord from the top of the snow stakes. B. The paracord threaded across the length and width of the fabric is anchored by the wooden dowels buried $1.2 \mathrm{~m}$ deep in the snow $\sim 1.5 \mathrm{~m}$ away from the fabric edge at a 45-degree angle. $\mathrm{C}$. The fabric is shown deployed flush to the snow surface.

F. Snow fabric Removal: During spring snowmelt

Remove the geotextile fabric. This should be when the manipulated snowmelt plot boundary is $60-$ $80 \%$ bare ground. Over the years of 2017-2019, the geotextile was removed within a date range of May 30 -June 7 to achieve $80 \%$ bare ground.

\section{Data analysis}

Visually over time, the induced snowmelt should be apparent from the time-lapse photos. The Onset Hoboware software downloadable on any computer will provide outputs of the hourly data during the experiment. It should be clear that the induced snowmelt plot has melted out before the paired control plot and it is possible to infer the time difference using the soil moisture and temperature results while visually validating dates with the time-lapse camera (Figure 5). 


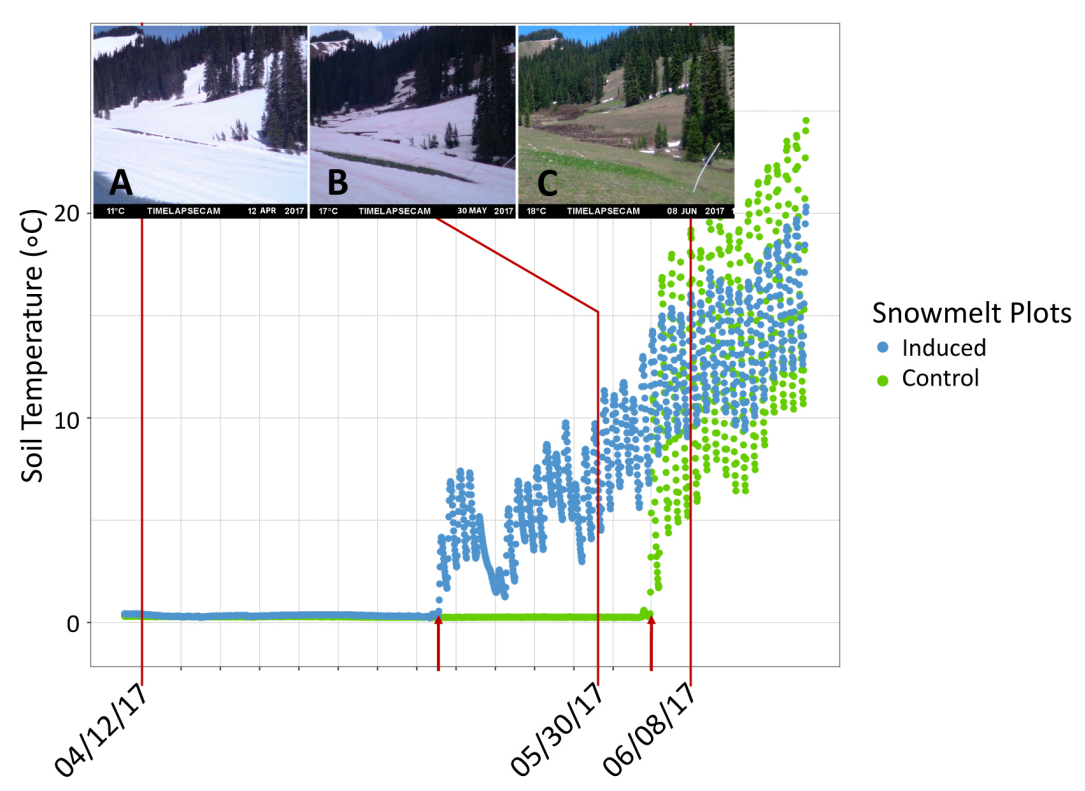

Figure 5. Data analysis to determine advanced snowmelt timing in 2017. Use of the time-lapse camera with the soil moisture temperature data will help determine the dates of full melt-out. The fabric was deployed on April $12^{\text {th }}(A)$. and melt-out of the induced plot appears to have occurred on May $13^{\text {th }}$ (first arrow) based on the soil temperature increase; however, the fabric was removed on May $30^{\text {th }}(B)$. New plant growth is apparent in the former location of the tarp demonstrating accelerated plant growth associated with snowmelt manipulation (C). Natural melt-out of the control plot began on June $4^{\text {th }}$ (second arrow), and the full control plot melted completely on June $8^{\text {th }}$ (C). It can be concluded from these observations that the induced snowmelt was advanced by three weeks.

\section{$\underline{\text { Notes }}$}

1. This method allows a variety of hydrological and ecological aspects of a plot-scale field experiment to be tested for additional analysis. Porewater can be collected using lysimeters; for example, in our experiment 20 lysimeters were utilized with a grid of $4 \times 5$ across each transect of the square plots. This value however is dependent on the experimental design and should be determined within the scope of each experiment. A minimum of six is suggested assuming three for the manipulated plot and control each. Soil microbial DNA analysis can also be conducted to study the soil microbial communities along with a variety of other methods easily adapted to this paired plot protocol.

2. When determining the experimental implementation of this protocol, it is recommended to consider whether environmental replicates of manipulated snowmelt are necessary for statistical analysis. Note that an increase in replicates will alter the number of materials and equipment needed, as the numbers provided here are for one manipulated plot and one control. An example of deployed fabric in triplicate is depicted in Figure 6. 


\section{bĭo-protocol

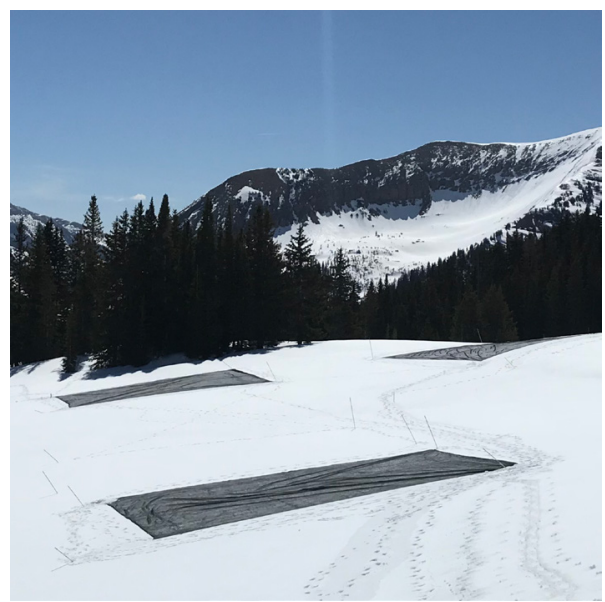

Figure 6. Deployed fabric in triplicate. An increase in replicates utilized for ongoing projects is displayed as an example if environmental replicates are deemed necessary.

3. Over the past three years using this method, the manipulated snowmelt plot has undergone advanced melt-out 14-23 days prior to the control. Use of the time-lapse camera allowed us to capture the behavior of the induced melting and determine when the complete melt-out of the manipulated versus the control plots occurred (Figure 7 and Video 1).

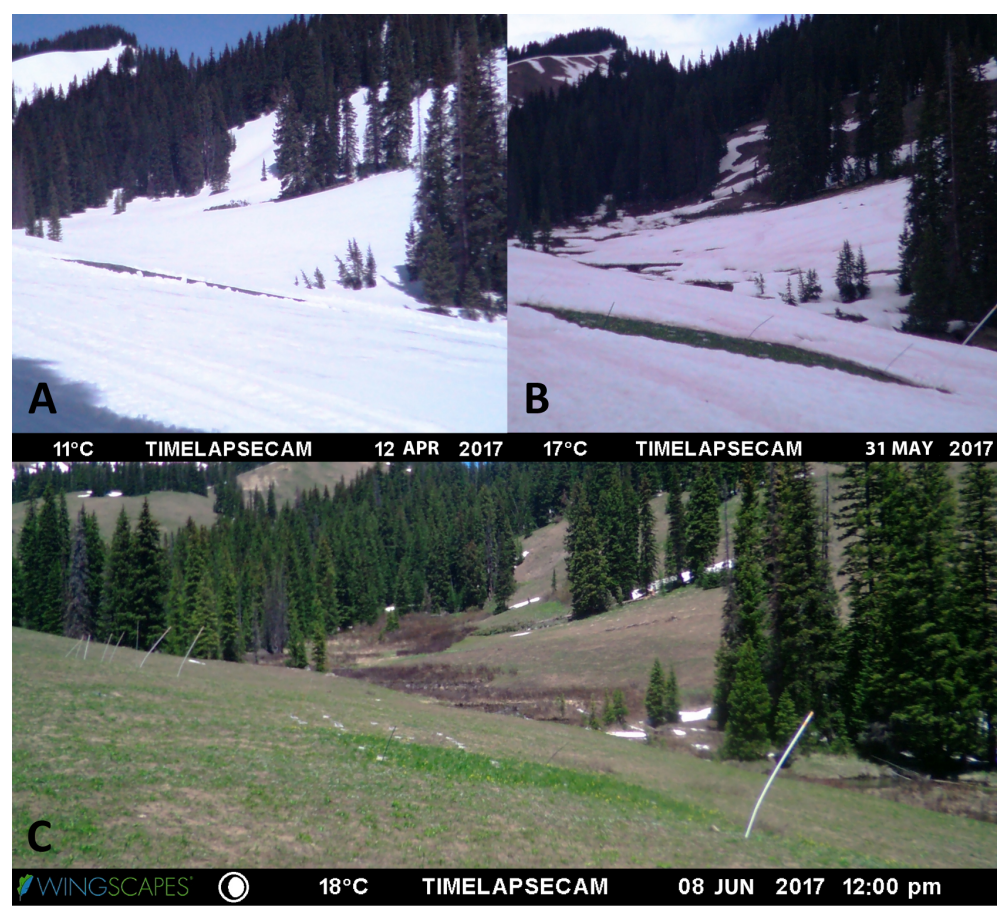

Figure 7. Time-lapse photos captured during induced snowmelt in 2017. Geotextile deployment on April $12^{\text {th }}(A)$, the fabric is removed on May $30^{\text {th }}(B)$, and full melt-out of the two paired plots on June $8^{\text {th }}(C)$. 


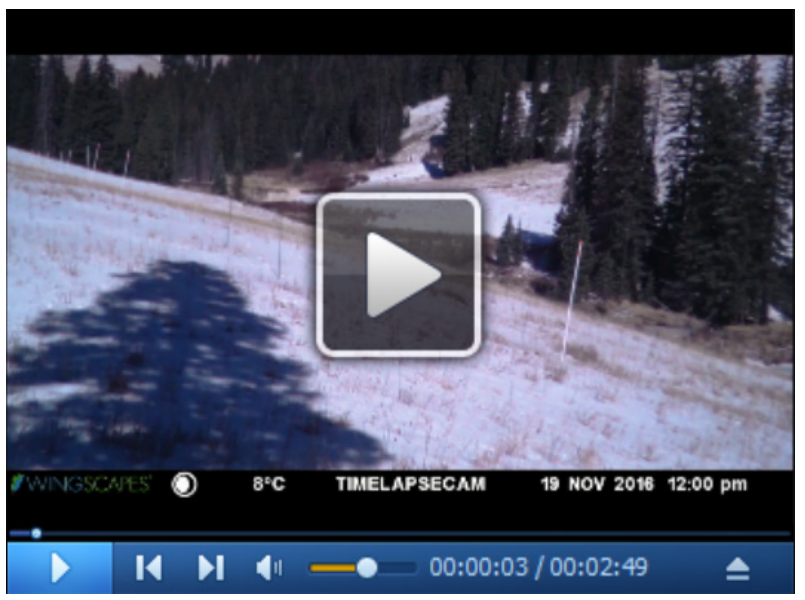

Video 1. Accelerated snowmelt time-lapse during 2016-2017 snow season

4. When deciding the best location for our study, we normalized the paired plots by choosing a meadow landscape with an east-northeast aspect to allow for sufficient sun irradiation throughout the day. Sun exposure is necessary for success of this protocol, which could complicate utility in forested regions. In addition, it is best to research annual snowfall depths and variability in the region of investigation to tailor deployment. Ensure the snow stakes will not be covered by the snowpack when it is time to deploy the geotextile. In addition, the snow stakes can partially bend in association with snowpack movement and wind on sloped surfaces effectively shortening their overall height. Create a contingency plan if the snow stakes are covered by the snowpack. We suggest mapping out the location measurements of the stakes using a long measuring tape and compass with respect to a non-moving landmark, or obtain precise GPS coordinates of the anchors.

5. Snow avalanche awareness is necessary for safe and successful implementation of the protocol. It is required in our research group that members trained in avalanche awareness help with deployment. Keep an eye on the conditions of your site and use avalanche reporting stations if available to analyze the risk. In addition, keep in mind that if the geotextile is deployed early, significant snowfall can upset the progress of the induced snowmelt by covering the fabric and returning sunlight absorption similar to that of the paired control. Advanced melting will not occur until the fabric is snow free, however in our experience subsequent snowfall has not strongly impacted the overall efficacy of this system with respect to net accelerated snowmelt.

\section{Acknowledgments}

Financial support was provided by the U.S. Department of Energy (DOE), Office of Science, Office of Biological and Environmental Research under exploratory university-led research: $D E-$ SC0016451 with partial support through the Lawrence Berkley National Laboratory's Watershed Function Scientific Focus Area under contract DE-AC02-05CH11231 (Lawrence Berkeley National Laboratory; operated by the University of California). Field access and support was provided by the 
Rocky Mountain Biological Laboratory (RMBL) in Gothic, CO. The Center for Snow and Avalanche Studies, Silverton, CO provided invaluable resources to test and implement the snowmelt approach used in the original implementation of this method. The authors thank Wendy Brown, Tony Brown, Amanda Henderson, and Kayla Hubbard for field and logistical support.

This protocol was adapted from previous work conducted by co-author Heidi Steltzer published in 2009, "Biological consequences of earlier snowmelt from desert dust deposition in alpine landscapes" (Steltzer et al., 2009).

\section{Competing interests}

Authors have no competing interest.

\section{$\underline{\text { References }}$}

1. Intergovernmental Panel on Climate Change (IPCC). (2014). AR5 Synthesis Report: Climate Change. https://www.ipcc.ch/report/ar5/syr/.

2. Barnett, T. P., Adam, J. C. and Lettenmaier, D. P. (2005). Potential impacts of a warming climate on water availability in snow-dominated regions. Nature 438 (7066): 303-9.

3. Blankinship, J. C., McCorkle, E. P., Meadows, M. W. and Hart, S. C. (2018). Quantifying the Legacy of snowmelt timing on soil greenhouse gas emissions in a seasonally dry montane forest. Global Change Biology 24 (12): 5933-47.

4. Bokhorst, S., Bjerke, J. W., Bowles, F. W., Melillo, J., Callaghan, T. V. and Phoenix, G. K. (2008). Impacts of extreme winter warming in the sub-arctic: growing season responses of dwarf shrub heathland. Global Change Biology 14 (11): 2603-12.

5. Clow, D. W., Williams, M. W. and Schuster, P. F. (2016). Increasing aeolian dust deposition to snowpacks in the Rocky mountains inferred from snowpack, wet deposition, and aerosol chemistry. Atmospheric Environment, Acid Rain and its Environmental Effects: Recent Scientific Advances, 146 (December): 183-94.

6. Demaria, E. M. C., Roundy, J. K., Wi, S. and Palmer, R. N. (2016). The effects of climate change on seasonal snowpack and the hydrology of the northeastern and upper midwest united states. Journal of Climate 29 (18): 6527-41.

7. Elias, E. H., Rango, A., Steele, C. M., Mejia, J. F. and Smith, R. (2015). Assessing climate change impacts on water availability of snowmelt-dominated basins of the upper rio grande basin. Journal of Hydrology: Regional Studies 3 (March): 525-46.

8. Harte, J., Torn, M. S., Chang, F., Feifarek, B., Kinzig, A. P., Shaw, R. and Shen, K. (1995). Global warming and soil microclimate: results from a meadow-warming experiment. Ecological Applications 5 (1): 132-50. 
9. Livensperger, C., Steltzer, H., Darrouzet-Nardi, A., Sullivan, P. F., Wallenstein, M. and Weintraub, M. N. (2016). Earlier snowmelt and warming lead to earlier but not necessarily more plant growth. AoB Plants 8: pii: plw021. https://doi.org/10.1093/aobpla/plw021.

10. Rauscher, S. A., Pal, J. S., Diffenbaugh, N. S. and Benedetti, M. M. (2008). Future changes in snowmelt-driven runoff timing over the western US. Geophysical Research Letters 35 (16). doi.org/10.1029/2008GL034424.

11. Steltzer, H., Landry, C., Painter, T. H., Anderson, J. and Ayres, E. (2009). Biological consequences of earlier snowmelt from desert dust deposition in alpine landscapes. Proc Nati Acad Sci U S A 106 (28): 11629.

12. de Vries, F. T. and Griffiths, R. I. (2018). Chapter 5: Impacts of Climate Change on Soil Microbial Communities and Their Functioning. In: Climate change impacts on soil processes and ecosystem properties. Developments in Soil Science. Horwath, W. and Kuzyakov, Y. (Eds.). Elsevier. 35:111-29.

13. Walsh, N. E., McCabe, T. R., Welker, J. and Parsons, A. (2003). Experimental manipulations of snow-depth: effects on nutrient content of caribou forage. Global Change Biology 3: 158-64.

14. Wipf, S., Rixen, C. and Mulder, C. P. H. (2006). Advanced snowmelt causes shift towards positive neighbour interactions in a subarctic tundra community. Global Change Biology 12 (8): 1496-1506. 